\title{
Welche medizinischen Thrombose- prophylaxestrümpfe - knielang oder oberschenkellang - sind effektiver?
}

\author{
V. Wienert ${ }^{1}$; M. Marshall ${ }^{2}$ \\ ${ }^{1}$ Universitätsklinikum Aachen; ${ }^{2}$ Privatpraxis Tegernsee
}

\begin{abstract}
Schlüsselwörter
Medizinischer Thromboseprophylaxestrumpf, Thromboseprophylaxe

Zusammenfassung

Ziel: Es sollte der präventive Effekt knielanger (Wadenstrumpf) und oberschenkellanger (Schenkelstrumpf) medizinischer Thromboseprophylaxestrümpfe (MTPS) evaluiert werden.

Methode: In den Datenbanken Pubmed, Cochrane Library, HTA-Berichte wurde nach den Stichwörtern "Thromboseprävention" und "Thromboseprophylaxestrümpfe" gesucht, und zwar in systematischen Reviews, Metaanalysen und Leitlinien.

Ergebnisse: Es finden sich zwei ergiebige Leitlinien und zwei sehr umfangreiche systematische Reviews, alle im Zeitraum von 2010 bis 2015 publiziert.
\end{abstract}

Die NICE-Leitlinie und die SIGN-Leitlinie empfehlen knielange oder oberschenkellange MTPS, vier Reviews dagegen zeigen keine eindeutigen Ergebnisse in der Prävention von Thrombosen.

Der präventive Effekt beider MTPS ist nicht deutlich unterschiedlich:

- Die Berechnung des präventiven Effekts beider MTPS ist nicht möglich, da die Studien signifikant heterogen sind.

- Der präventive Effekt des oberschenkellangen MTPS, kombiniert mit der Pharmakotherapie, zeigt einen nicht signifikanten positiven Trend.

- Der präventive Effekt eines oberschenkellangen MTPS plus Pharmakotherapie ist effektiver als der eines knielangen MTPS plus Pharmakotherapie.

\section{Keywords}

Anti-embolism stocking, thromboprophylaxis

Summary

Aim: The preventive effect of knee-length and thigh-length anti-embolism stockings should be evaluated.

Method: We searched in PubMed, CochraneLibrary and Health Technology Assessment for guidelines, systematic reviews and metaanalyses.

Results: The NICE-Guideline recommends thigh-length or knee-length anti-embolism stockings for prevention of deep venous thrombosis. The reviews show no significant different effects of the two kinds of anti-embolism stockings
Korrespondenzadresse

Prof. Volker Wienert

Hautklinik

Universitätsklinikum Aachen

Tel. 0241/8080832

E-Mail: avwienert@gmail.com

\section{Zitierweise des Beitrages/Cite as:}

Which kind of anti-embolism stockings - up to knee or up to thigh - are more effective?

Phlebologie 2018; 47: 13-15

https;//doi.org/10.12687/phleb2395-1-2018

Eingereicht: 17. November 2017

Angenommen: 04. Dezember 2017

English version available at:

www.phlebologieonline.de
In der Allgemeinbevölkerung liegt die jährliche Inzidenz der symptomatischen tiefen Venenthrombose (TVT) im Mittel bei $0,1 \%$ (1). Die TVT kann zu einer Lungenembolie oder später auch zu einem postthrombotischen Syndrom führen. Im Krankenhaus beträgt die peri- und postoperative TVT-Rate ohne prophylaktische Maßnahmen je nach Eingriff 0,2 bis $50 \%$ $(\checkmark$ Tab. 1). Neben dem Alter bedeuten auch andere Faktoren für den Patienten ein erhebliches Risiko. So trägt z.B. eine gravide Frau ein zehnfaches und ein Karzinomträger ein fünf- bis siebenfaches Risiko ( Tab. 2). Da im Krankenhaus nicht nur eine Operation sondern auch die nachfolgende Immobilisation des Patienten mit einem Thromboserisiko einhergeht, ist hier eine Prävention der TVT durch das Tragen von medizinischen Thromboseprophylaxe- strümpfen (MTPS) und evtl. einer zusätzlichen Antikoagulation angeraten.

Die Indikationen für den MTPS sind folgende:

- prä-, intra- und postoperative Thromboseprophylaxe,

- peri- und postpartale Thromboseprophylaxe,

- generelle Thromboseprophylaxe bei bettlägerigen Patienten. 
Folgende Kontraindikationen müssen beachtet werden:

- fortgeschrittene periphere arterielle Verschlusskrankheit,

- Phlegmasia coerulea dolens, septische Phlebitis,

- dekompensierte Herzinsuffizienz,

- Dermatitis, Ulcus cruris.

Die MTPS sind aus Polyamid und Elastan gefertigt und unterliegen, im Gegensatz zu den medizinischen Kompressionsstrümpfen (MKS), keiner Norm. So werden MTPS je nach Fabrikat in 5 bis zu 40 unterschiedlichen Größen, in verschiedenen Längen (knielang, oberschenkellang) und mit uneinheitlicher Elastizität (nur querelastisch oder querelastisch/längselastisch) angeboten.

Der Druck des MTPS im Knöchelbereich beträgt in der Regel $18 \mathrm{mmHg}$. Die Patienten präferieren knielange MTPS. Bei Durchsicht der Literatur fällt auf, dass der Begriff MTPS in den verschiedenen Publikationen mit differenten Namen belegt ist, z.B. mit Antiembolism stockings (AES), Antithrombosestrümpfe (ATS), auch graduated compression stockings (GCS) oder deterrent stockings. Der Begriff GCS sollte hier gar nicht geführt werden, da er bereits synonym ist mit dem medizinischen Kompressionsstrumpf (MKS).

\section{Wirkung}

Mittels objektiver Messmethoden wurde die positive Wirkung des MTPS auf die untere Extremität nachgewiesen. Bei bettlägerigen Patienten kommt es infolge eines vergrößerten Venenquerschnitts durch Tonusverlust und Ausfall der Waden- und Sprunggelenkpumpe zu einer beachtlichen venösen Strömungsverlangsamung. Der Andruck des MTPS auf das Bein erhöht den Gewebedruck, reduziert den Venenquerschnitt, steigert somit den Abfluss und reduziert das Blutvolumen im Bein (Lawrence et al., Sigel et al., Sparrow et al.).

\section{Methodik}

Es wurde in den Datenbanken Pubmed, Cochrane Library, Health Technology As-
Tab. 1 Postoperative TVT bei fehlenden prophylaktischen Maßnahmen (nach [8])

\begin{tabular}{l|l}
\hline Art des Eingriffes & TVT (\%) \\
\hline Größere abdominelle OP & $20-40$ \\
\hline Gefäß-OP (z.B. Aorta) & $20-27$ \\
\hline $\begin{array}{l}\text { Hüftgelenk-Kniegelenk- } \\
\text { OP }\end{array}$ & 50 \\
\hline Mamma-Ca-OP & $5-20$ \\
\hline Thorax-OP & $9-18$ \\
\hline Neurochirurg.-OP & $20-35$ \\
\hline Größere Wirbelsäulen-OP & $0,3-2,2$ \\
\hline Varizen-OP & 0,2 \\
\hline Urologische OP & bis 30 \\
\hline
\end{tabular}

sessment systematisch nach „Thromboseprävention“, „medizinische Thromboseprophylaxestrümpfe knielang, oberschenkellang" gesucht, und zwar nach systematischen Reviews, Metaanalysen und Leitlinien.

\section{Ergebnisse}

Es fanden sich zwei entsprechende Leitlinien und zwei umfangreiche systematische Reviews, alle im Zeitraum 2010 bis 2015 publiziert.

In den von uns berücksichtigten zwölf Leitlinien wird in zwei Ausgaben der MTPS nicht erwähnt $(3,6)$.

Die NICE-Leitlinie (2010) des Institute for Health and Care Excellence (7) empfiehlt oberschenkellange oder knielange MTPS, oft auch kombiniert mit einer Anti-

\section{Tab. 2 Risikofaktoren einer TVT (SIGN)}

\begin{tabular}{l|l}
\hline Gravidität & 10-faches Risiko \\
\hline Adipositas & 2-3-fach \\
\hline Karzinom & 5-7-fach \\
\hline Orale Kontrazeption & 3-6-fach \\
\hline Varizen & 1,5-2,5-fach \\
\hline
\end{tabular}

Ein weiterer wesentlicher Risikofaktor ist eine stattgehabte TVT. Der größte Risikofaktor ist jedoch das Alter. Die jährliche Inzidenz der TVT von Personen jünger als 40 Jahre beträgt 1:10.000, die der 60-69-Jährigen 1:1.000 und die der älter als 80-jährigen Personen 1:100 (10). koagulation, für immobile Patienten mit den Eingriffen:

- Herzoperationen

- Gastroenterologische, gynäkologische, thorakale und urologische Operationen

- Neurochirurgische Eingriffe (kranial, spinal)

- Hüftgelenksersatzoperationen

- Kniegelenksersatzoperationen

- Operation der Hüftgelenksfraktur

- Gefäßoperationen

- Eingriffe nach größeren Verletzungen, auch der Wirbelsäule

Außerdem empfiehlt diese Leitlinie die Anwendung von MTPS oberschenkellang oder knielang auch bei Patienten mit internistischen Erkrankungen, wenn die Pharmakoprophylaxe nicht indiziert ist.

Diese Leitlinie empfiehlt nicht die Anwendung der MTPS bei Patienten mit Schlaganfall.

\section{SIGN-Leitlinie (2014) (Scottish Intercollegiate Guidelines (10)}

Den meisten randomisierten kontrollierten Studien (RCTs) zufolge werden oberschenkellange MTPS zur Prävention eingesetzt.

Die Anzahl der RCTs, die den knielangen MTPS thematisieren, ist zu gering, um den Effekt dieser Strümpfe ermitteln zu können. In einer Metaanalyse kommt man zu dem Schluss, dass es keine größeren Unterschiede hinsichtlich des Effektes bei chirurgischen Patienten gibt.

Diese Leitlinie empfiehlt deshalb, knielange oder oberschenkellange MTPS sollten zur Prophylaxe der TVT bei chirurgischen Patienten eingesetzt werden, wenn es keine Kontraindikationen gibt und die Strümpfe korrekt sitzen.

Gemäß den von uns durchgesehenen zehn systematischen Reviews ist der MTPS bei differenten Eingriffen indiziert, manchmal in Kombination mit der Pharmakotherapie.

\section{Cochrane Library (2012) (2)}

In diesem systematischen Review werden drei randomisierte kontrollierte Studien mit insgesamt 496 Patienten ( $\mathrm{n}=201$ knielange MTPS, $n=295$ oberschenkellange MTPS) ausgewertet. Die Resultate zeigen 
keinen signifikanten Unterschied in der Reduktion der postoperativen TVT. Knielange MTPS waren gleich effektiv wie die oberschenkellangen MTPS hinsichtlich der Thromboseprophylaxe. Nach Ansicht der Autoren muss das Fazit als nicht aussagekräftig angesehen werden, da die Studien eine deutliche Heterogenität aufweisen, sowohl hinsichtlich der Randomisierungstechnik als auch der statistischen Power und der Intention-totreat-Analyse.

\section{Health Technology Assessment (2015) (4)}

Es handelt sich um ein systematisches Review (219 Seiten), in dem die Daten von fünf RCT's gepoolt wurden, um den Effekt von knielangen $(n=359)$ und oberschenkellangen MTPS ( $\mathrm{n}=590)$, oft kombiniert auch mit der Pharmakotherapie, vergleichen zu können. Ermittelt wurde ein nicht signifikanter positiver Trend für den oberschenkellangen MTPS (Odds Ratio 1,48).

Eine Netzwerk-Metaanalyse von 13 RCTs ( $n=3691)$ ergab eine größere Effektivität des oberschenkellangen MTPS, kombiniert mit einer Pharmakotherapie, als die Kombinationstherapie knielanger MTPS (Odds Ratio 1,76).

\section{Diskussion}

Die Reviews zeigen hinsichtlich des präventiven Effekts von knielangen MTPS und oberschenkellangen MTPS kein eindeutiges Ergebnis. Dies ist gut erklärbar: In den Studien wurden den Patienten sog. EinzugMTPS (nur querelastisch) als auch Zweizug-MTPS (quer- und längselastisch) verabreicht. Im Gegensatz zum Einzug-MTPS hat der Zweizug-MTPS bei unterschiedlicher Längsdehnung auch eine unterschiedliche Querdehnung und kann somit den abgestuften Kompressionsdruck an definierten Stellen des Beins kaum gewährleisten.

Außerdem wurden in den Studien MTPS von verschiedenen Herstellern verwendet, die je nach Produkt von 5 bis zu 40 unterschiedlichen Größen anbieten. Ein Produkt, das in weniger als 10 Größen hergestellt wird, kann nicht den Bedarf der gesamten Bevölkerung abdecken. Zudem sind der Druck wie auch der Druckverlauf der verschiedenen MTPS nicht einheitlich. Die genannten Faktoren mindern die Aussagekraft der zitierten systematischen Reviews.

\section{Interessenkonflikt}

Nach Angaben der Autoren bestehen keine Interessenkonflikte.

\section{Ethische Richtlinien}

Für das Manuskript wurden keine Studien an Menschen oder Tieren durchgeführt.

\section{Literatur}

1. AWMF-S3-Leitlinie. Prophylaxe der venösen Thromboembolie. AWMF-de 2015.
2. Cochrane Library. Kneelength versus thigh length graduated compression stockings for prevention of deep vein thrombosis in postoperative surgical patients. 2012.

3. Falck-Ytter Y, Francis CW, Johanson NA et al. Prevention of VTE in orthopedic surgery patients. Antithrombotic therapy and prevention of thrombosis. 9th ed. American College of Chest Physicians Evidence-based clinical practice guidelines. Chest 2012; 141 (2) Suppl: e278S-e325S.

4. Wade R, Sideris E, Paton F et al. Graduated compression stockings for the prevention of deep-veinthrombosis in postoperative surgical patients: a systematic review and economic model with a value of information analysis. Health Technology Assessment 2015; 19.

5. Lawrence D, Kakkar VV. Graduated, static, external compression of the lower limb: a physiological assessment. Br J Surg 1980; 67: 119-121.

6. Monagle P, Chan HKS, Goldenberg NA et al. Antithrombotic therapy in neonates and children. Antithrombotic therapy and prevention of thrombosis. 9th ed.: American College of Chest Physicians. Evidence-based clinical practice guidelines. Chest 2012; 141 (2) Suppl: e737S-e801S.

7. NICE-Guideline (2010) National Institute for Health and Care Excellence. Using VTE prophylaxis.

8. Samama CM, Albaladejo P, Benhamou D et al. Venous thromboembolism prevention in surgery and obstetrics: clinical practice guideline. Eur J Anaesthes 2006; 28: 95-116.

9. Sigel M, Edelstein AL, Savitch L et al. Type of compression for reducing venous stasis: A study of lower extremities during inactive recumbency. Arch Surg 1975; 110: 171-175.

10. SIGN-Guideline (2014) Scottish Intercollegiate Guidelines: Prevention and management of venous thromboembolism.

11. Sparrow RA, Hardy JG, Fentem PH. Effect of antiembolism compression hosiery on the leg blood volume. Br J Surg 1995; 82: 53-59. 